\title{
FDA approval of Pfizer-BioNTech: Evaluating the significance of mRNA vaccine approval
}

\author{
Syed Abdullah Monawwer ${ }^{1}$, Raahim Naeem ${ }^{2}$ \\ ${ }^{1}$ Internal Medicine, Ziauddin Medical University, ${ }^{2}$ Internal Medicine, Dow University of Health Sciences \\ Keywords: Vaccine, COVID-19, BioNTech, Pfizer, FDA, Comirnaty, mRNA vaccine, SARS-CoV-2, Pandemic \\ https://doi.org/10.52872/001c.30733
}

\section{Journal of Global Health Economics and Policy}

Vol. 1, 2021

\begin{abstract}
The COVID-19 pandemic has devastated the global demographics, claiming more than 4.8 million deaths globally as of October 2021. Mass vaccination is currently the best defense strategy against the virus, which has led to efforts towards the global distribution of COVID-19 vaccines. However, due to the urgency to inoculate, not all vaccines had received federal endorsement by the Food and Drug Administration (FDA) of the United States of America. The FDA is widely considered one of the foremost authorities on drug safety, which is why their ratification of an mRNA vaccine produced by Pfizer, Inc. and BioNTech is a landmark for vaccinology. Although the vaccine was already in distribution, vaccine hesitancy was exacerbated by the lack of timely FDA approval, resulting in the overall lower than expected vaccination rates. Another detrimental factor was distrust in COVID-19 vaccines, based on the widespread claims of adverse effects, despite the vaccine safety being demonstrated in multiple studies. Not only are these new vaccines on a pace to outperform the traditional attenuated virus vaccines, their underlying technology and the use of the RNA molecule ushers in a new era, in which vaccines can be applied and adjusted quicker and for a broader spectrum of targets.
\end{abstract}

The Food and Drug Administration (FDA) of the United States of America passed a landmark decision to approve Pfizer and BioNTech's vaccine usage and distribution on the $23^{\text {rd }}$ of August, 2021. The vaccine will be marketed as Comirnaty and distributed to all individuals aged 16 and older. ${ }^{1}$ This decision reflects the necessity of vaccination in the United States, which has had more than 43 million cases and upwards of 700,000 deaths due to Covid-19 as of October $2021 .^{2}$ The Pfizer-BioNTech vaccine is one of the several vaccines currently distributed in the United States, although it is the first Covid-19 vaccine to get approval from the FDA. The need for vaccines to combat the severe acute respiratory syndrome coronavirus-2 (SARS-CoV-2) has led to a massive acceleration in the manufacture of vaccines like Comirnaty. Boosting the production of such vaccines has been instrumental in flattening the curve of viral transmissions and preventing hospital facilities from getting overwhelmed. This document aims to discuss the numerous factors that led to the approval of Pfizer-BioNTech's new vaccine and the importance of this approval by the FDA.

The vaccine had been available for public distribution since the $10^{\text {th }}$ of December, 2020; due to the severity of the Covid-19 pandemic, the need for FDA approval prior to distribution was waived. In some cases, such as individuals below the age of 16 and above 12, special provisions were made in the form of EUAs (Emergency Use Authorizations). Through EUAs, many vaccines have been made available and resulted in millions of vaccinations from the middle of 2020 to date. In the United States, an estimated 215 million individuals have been fully vaccinated as of October 2021 , attributing to about $65 \%$ of the populace. ${ }^{2}$ This reflects a lower total percentage population than countries of similar economic standing, such as OECD nations, where the percentage of the vaccinated population can be as high as $94 \% .^{3}$

Various political and social elements explain this disparity - vaccine hesitancy is a major one. This is exacerbated by distrust of the vaccine's reliability, as the FDA had not previously approved any vaccines directly. Pfizer-BioNTech's vaccines breaking that trend will undoubtedly result in much higher vaccination rates as a result. Vaccines like Comirnaty, mRNA vaccines, have been the subject of much debate. However, in 2020, following the rapid spread of SARS-CoV-2, we have seen a rapid acceleration of mRNA vaccine production and heavy investment into their development. Due to necessity and because the technology had just been perfected, mRNA vaccines were among the first to begin distribution. The efficacy of such vaccines has been observed to be significantly higher than that of traditional vaccines made from attenuated viruses. One study was able to show that under observation, the vaccine protected individuals from COVID-19 in $95 \%$ of cases. ${ }^{4}$ Vector vaccines resulted in lower protection rates, mostly around 70\%; the Pfizer-BioNTech vaccine can be seen to provide more consistent protection from COVID-19.4 It must also be noted that the vaccine's efficacy cannot be judged by clinical trials alone. The nature of the vaccine itself further complicates the matter, as there is evidence to support the idea that mRNA vaccines may not result in the same linear rate of vaccine efficacy decline that other types of vaccines are subject to; in fact, the opposite was noted by a study. ${ }^{5}$ This paper alludes to the fact that immunologically, plasma neu- 
tralizing antibodies do decline after several months of vaccination. However, this effect is possibly eclipsed by the fact that the cells responsible for creating those antibodies, the memory B cells, keep a healthy population count. Moreover, these memory B cells, which are measures of the body's immune response against Covid-19, are found to increase in number and efficacy over at least six months, even against different variants of viruses. This means it is not directly possible to quantify the extent of the protection of mRNA vaccines like Comirnaty without extensive research - however, preliminary and theoretical evidence suggests that the duration of protection of Comirnaty might be much longer than previously expected. ${ }^{5}$

As mRNA vaccines develop, the time to manufacture each subsequent vaccine is expected to decrease. While speaking at a press event in December, Uğur Şahin, the CEO of BioNTech stated that an mRNA vaccine could be synthesized in as little as a matter of weeks. Moreover, the vaccine was able to demonstrate its safety as well. As found by one study, the vaccine was proven to be safe. ${ }^{6}$ Out of a sample of 43,548 , of which 43,448 received injections $(21,720$ received Pfizer-BioNTech mRNA vaccines and 21,728 received placebo injections), only 8 cases of Covid-19 were observed amongst the group receiving the vaccine. In the control group, there were 162 cases of Covid-19. Furthermore, of the ten severe COVID-19 cases, nine occurred from within the placebo group. This study and others are somewhat limited in scope, but are generally accepted as evidence that the Pfizer-BioNTech vaccine is safe for public usage. ${ }^{6}$

Comirnaty, as any other mRNA vaccine, acts to provide immunity in a very simple manner. A surface protein on the SARS-CoV-2 virus is extracted. This protein resembles a spike in shape and is therefore referred to as such. This protein is synthesized from mRNA, a single strand of nucleotides that encode polypeptides. By deducing the sequences of amino acids that make up the 'spike' protein on SARS-CoV-2, researchers were able to understand exactly the composition of the protein, and thereby reverse engineer it. As the mRNA is taken up by the cell, the Rough Endoplasmic Reticulum (RER) synthesizes the spike protein. The mRNA does not frequently enter the nucleus of the cell, contrary to popular misconception. The mass production of the spike protein allows for high concentrations of the antigen, resulting in a stronger immune response against SARSCoV-2. Furthermore, this method of inoculation carries less risk of complications from the vaccine, especially as there is no risk of infection or insertional mutagenesis, which are known risks of using live-attenuated vaccines. ${ }^{7}$

The Comirnaty vaccine is known to be associated with some side effects. These commonly include mild conditions, such as soreness, fatigue, myalgia, and headache. More severe conditions were found to be extremely rare. ${ }^{8}$ The FDA's approval of Comirnaty was contingent upon Pfizer and BioNTech providing sufficient evidence for its safety. Over the course of the year during which Comirnaty was under review, evidence has shown by and large that the potential adverse effects of the vaccine are rare, and the vaccine is safe on the whole. However, there were also widespread concerns about this vaccine when it was reported early on that Comirnaty was strongly linked with anaphylaxis, which was widely discussed in the medical commu- nity. ${ }^{9}$ These concerns led to increased caution before administering the vaccine in those suspected to be more susceptible to allergenic hypersensitivity.

In some cases, individuals were even discouraged openly from using this vaccine if they were at risk of strong anaphylaxis especially to the vaccine ingredient PEG (polyethylene glycol), by the CDC. ${ }^{10}$ Due to these measures taken early on, the incidence of severe adverse reactions to the Pfizer-BioNTech vaccines was mitigated. Still, it bears further investigation into the possible adverse effects the vaccine may have on a select few individuals. However, this risk was pointed out to not be inherent only to the vaccine, as we have discussed earlier, but rather a possible, albeit rare, complication of all forms of inoculation.

The importance of this safety certification by the FDA is yet to be seen. However, it is speculated that the approval will help persuade many Americans to get vaccinated. In urban areas, many individuals who have access to vaccination programs were skeptical of the vaccine's safety due to political and social factors. A recent poll by the Kaiser Family Foundation found that among those who were hesitant to get inoculated against SARS-CoV-2, about 3 in 10 said they would get vaccinated if the FDA approved the vaccines. ${ }^{11}$ However, many remain unsure whether the FDA approval alone will be sufficient to convince the majority of the unvaccinated, primarily due to the politicization of the issue. The extent of the division of vaccinated and unvaccinated individuals among party lines has been debated extensively, without much statistical evidence to settle the matter. Furthermore, the variance in geopolitical factors for vaccine hesitancy means that it is impossible to say whether the same hesitancy will be seen globally - in fact, this notion is almost entirely ungrounded in data. Diverse locations naturally must have different objections to the vaccine, or none at all, and it is essential to await further data before proposing that attitudes towards inoculation might change.

It can be noted that in underdeveloped and developing nations, the spread of COVID-19 has been exacerbated by delays in acquiring vaccines. In particular, impoverished nations like Pakistan have been hit especially hard by the coronavirus pandemic, and their response revolved entirely around acquiring vaccines. However, experts have opined that the minor delay of a few months after the release of the vaccines and their arrival in Pakistan has been devastating for the country. ${ }^{12}$ Moreover, it is expected that the inequity in vaccination might lead to a net loss of $\$ 153$ billion a year in GDP, according to a report by RAND Europe. ${ }^{13}$ Of course, it would be negligent to discuss the implications of worldwide pandemics and restrict the discussion to but a few nations. The most threatening concern to worldwide health in recent times has been a surge in viral mutations, leading to a plethora of Coronavirus variants. One of the biggest causes of such mutations has been claimed to be the large pool of unvaccinated individuals, in which viruses with individual mutations may intermix, leading to the birth of new strains of SARS-CoV-2. The severity of this threat to global health cannot be understated. It may be that until vaccine inequality ends, and the total population of the world receives adequate inoculation, the pandemic may never end. However, it must also be clarified that the 
situation is evolving, and it is impossible to weigh the effects of vaccine unavailability until further data is compiled.

Nonetheless, as the technology to develop these vaccines improves with funding and grants, mRNA vaccines could eventually outpace live-attenuated vaccines in production and safety. Pfizer-BioNTech's vaccine has solved some of the many problems that plagued early mRNA vaccines, such as rapid decomposition of the vaccine when exposed to outside conditions. The new vaccine can remain stable and usable inside the temperature range of -25 degrees Celsius to -15 degrees Celsius, which can be ensured by a household refrigerator, for up to 2 weeks, whereas previous vaccines using mRNA could become unfit for usage if not kept below -60 degrees Celsius. ${ }^{14}$ Moreover, the vaccine has ushered in a new era of modified nucleoside mRNA vaccines. These vaccines use a modified mRNA nucleoside, such as pseudouridine, instead of the naturally occurring counterpart: in this case, uridine. For decades, experts debated which base was more effective, but using a modified nucleoside in Comirnaty and the success the vaccine has found will pave the way for more modified nucleosides to be used in these vaccines.

In conclusion, the approval of Pfizer-BioNTech's Comirnaty vaccine is a landmark accomplishment for genetic engineering as a field and medicine on the whole. The approval will help mRNA vaccines gain authenticity and benefit millions, if not billions, in protecting individuals from COVID-19. It can also be said that through this approval, a host of competitor brand mRNA vaccines will also vie for market share, allowing vaccines to be available to more people across the globe. Moreover, the vaccine represents the culmination of decades of research and presents a new alternative to traditional vaccination. By recognizing the importance of such research and ensuring the products meet rigorous safety criteria before approval, the FDA has shown its faith in emerging technologies yet kept a firm commitment to protecting people's health.

\section{FUNDING}

None

\section{ACKNOWLEDGEMENTS}

None

\section{COMPETING INTERESTS}

The authors completed the ICMJE Unified Competing Interest form at (available upon request from the corresponding author), and declare no conflicts of interest.

\section{AUTHOR'S CONTRIBUTIONS}

Both authors participated in all stages of the manuscript development and drafting. In addition, both authors provided critical feedback on the manuscript and have agreed to be accountable for all aspects of the work.

\section{CORRESPONDENCE}

Syed Abdullah Monawwer, Department of Internal Medicine, Ziauddin Medical University, 4/B Shahrah-e-Ghalib Rd, Block 6 Clifton, Karachi, Sindh, Pakistan; phone: +92-3350364583; email: abdullah.munawwer@gmail.com

Submitted: November 22, 2021 CEST, Accepted: December 02, 2021 CEST 


\section{REFERENCES}

1. Office of the Commissioner. FDA Authorizes Booster Dose of Pfizer-BioNTech COVID-19 Vaccine for Certain Populations.https://www.fda.gov/news-ev ents/press-announcements/fda-authorizes-booster-d ose-pfizer-biontech-covid-19-vaccine-certain-popula tions. Published September 22, 2021. Accessed December 2, 2021.

2. CDC. COVID Data Tracker. Published March 28 , 2020. Accessed December 2, 2021. https://covid.cdc.g ov/covid-data-tracker

3. Ritchie H, Mathieu E, Rodés-Guirao L, et al. Coronavirus Pandemic (COVID-19). Our World in Data.https://ourworldindata.org/coronavirus. Published March 5, 2020. Accessed December 2, 2021.

4. Okamura S, Ebina H. Could live attenuated vaccines better control COVID-19? Vaccine.

2021;39(39):5719-5726. doi:10.1016/j.vaccine.2021.0 $\underline{8.018}$

5. Scott J, Richterman A, Cevik M. Covid-19 vaccination: evidence of waning immunity is overstated. BMI. 2021;374. doi:10.1136/bmi.n2320

6. Polack FP, Thomas SI, Kitchin N, et al. Safety and Efficacy of the BNT162b2 mRNA Covid-19 Vaccine. $N$ Engl J Med. 2020;383(27):2603-2615. doi:10.1056/nej $\underline{\text { moa2034577 }}$

7. Pardi N, Hogan MJ, Porter FW, Weissman D. mRNA vaccines - a new era in vaccinology. Nat Rev Drug Discov. 2018;17(4):261-279. doi:10.1038/nrd.2017.243

8. Baden LR, El Sahly HM, Essink B, et al. Efficacy and Safety of the mRNA-1273 SARS-CoV-2 Vaccine. $N$ Engl J Med. 2021;384(5):403-416. doi:10.1056/nejmoa $\underline{2035389}$
9. Kadali RAK, Janagama R, Peruru S, Malayala SV. Side effects of BNT162b2 mRNA COVID-19 vaccine: A randomized, cross-sectional study with detailed selfreported symptoms from healthcare workers. Int J Infect Dis. 2021;106:376-381. doi:10.1016/j.ijid.2021.0 $\underline{4.047}$

10. Kim MA, Lee YW, Kim SR, et al. COVID-19 Vaccine-associated Anaphylaxis and Allergic Reactions: Consensus Statements of the KAAACI Urticaria/Angioedema/Anaphylaxis Working Group. Allergy, Asthma \& Immunology Research. 2021;13(4):526-544. doi:10.4168/aair.2021.13.4.526

11. KFF COVID-19 Vaccine Monitor: Parents and the Pandemic. KFF. https://www.kff.org/coronavirus-covi d-19/poll-finding/kff-covid-19-vaccine-monitor-pare nts-and-the-pandemic/. Published August 11, 2021. Accessed December 12, 2021.

12. Kamran K, Ali A. Challenges and Strategies for Pakistan in the Third Wave of COVID-19: A Mini Review. Frontiers in Public Health. 2021;9. doi:10.338 9/fpubh.2021.690820

13. Asundi A, O'Leary C, Bhadelia N. Global COVID-19 vaccine inequity: The scope, the impact, and the challenges. Cell Host \& Microbe. 2021;29(7):1036-1039. doi:10.1016/j.chom.2021.06.00 $\underline{7}$

14. Comirnaty (COVID-19 Vaccine, mRNA) 11 Storage, Stability And Disposal. Published December 12, 2021. https://www.pfizermedicalinformation.ca/e n-ca/pfizer-biontech-covid-19-vaccine/11-storage-st ability-and-disposal 